\title{
Compositional and Gate Tuning of the Interfacial Conductivity in $\mathrm{LaAlO}_{3} / \mathrm{LaTiO}_{3} / \mathrm{SrTiO}_{3}$ Heterostructures
}

\author{
Masayuki Hosoda, ${ }^{1,2, *}$ Christopher Bell, ${ }^{1}$ Yasuyuki Hikita, ${ }^{1}$ and Harold Y. Hwang ${ }^{1,3}$ \\ ${ }^{1}$ Stanford Institute for Materials and Energy Sciences, \\ SLAC National Accelerator Laboratory, Menlo Park, CA 94025, USA \\ ${ }^{2}$ Department of Advanced Materials Science, The University of Tokyo, Kashiwa, Chiba 277-8561, Japan \\ ${ }^{3}$ Geballe Laboratory for Advanced Materials, Department of Applied Physics, Stanford University, Stanford, CA 94305, USA
}

We investigate the effect of $\mathrm{LaTiO}_{3}$ insertion at the interface between $\mathrm{LaAlO}_{3}$ and $\mathrm{TiO}_{2}$ terminated $\{100\} \mathrm{SrTiO}_{3}$, for a series of $\mathrm{LaAlO}_{3}$ and $\mathrm{LaTiO}_{3}$ thicknesses. A clear increase of the carrier density was observed while the Hall mobility was largely unchanged. In structures with $\mathrm{LaAlO}_{3}$ thickness $\sim 3$ unit cells, close to the critical thickness for conductivity, as little as 0.25 unit cells of $\mathrm{LaTiO}_{3}$ drives an insulator-to-metal transition. These samples show a strong dependence of the conductivity on voltage with electrostatic back-gating, which can be understood in a two-carrier picture, and dominated by the change in carrier density at the interface.

The $\mathrm{LaAlO}_{3} / \mathrm{SrTiO}_{3}(\mathrm{LAO} / \mathrm{STO})$ conducting interface is generating intense interest because of the highly mobile electrons present within a nanoscale thickness in the STO.[1] At the interface twodimensional (2D) superconductivity,[2] 2D Shubnikov-de Haas oscillations, [3, 4] and magnetism, [5-7] have all been observed. Although the relative contributions of oxygen vacancies, [8-10] interdiffused dopants, [11] and the polar discontinuity (PD), $[1,12-15]$ to these properties are still being debated, an intriguing aspect of the PD model is the possibility of modulation doping the STO, leading to relatively high Hall mobility due to the absence of scattering from ionized dopants.

This system is particularly interesting since close to the critical thickness of LAO required for conductivity $[\sim 3$ unit cells (uc)], a back gate voltage applied on the STO can induce conductivity in an as-grown insulating sample at room temperature.[13] Back gating strategies have been used to tune the $2 \mathrm{D}$ superconductivity at low temperatures.[16] A disadvantage of gating is that the sheet carrier density $n_{2 \mathrm{D}}$ is not modulated independently of the Hall mobility $\mu$.[17] In order to fully explore the phase diagram of the LAO/STO system, other techniques to control $n_{2 \mathrm{D}}$ are needed. Chemically doped STO layers, inserted between the LAO and undoped STO substrate, have been utilized to achieve this aim.[18-20]

In the context of these open questions, the

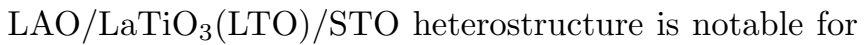
several reasons. Given the stacking structure of $A B \mathrm{O}_{3}$ in the $\{100\}$ planes, two independent interfaces can be defined, corresponding to the positions at which the chemical make-up of the $A \mathrm{O}$ and $B \mathrm{O}_{2}$ planes switch, as sketched in Fig. 1(a). The inserted LTO layer(s) spatially separates these $A$-site and $B$-site interfaces in a structurally clean manner, exploiting the close lattice match to STO. Simultaneously LTO can provide carrier modulation without placing dopant ions at the interface, since it contains a high density of electrons: for each layer of LTO, there exists a potential sheet carrier density of
$6.5 \times 10^{14} \mathrm{~cm}^{-2}$ per lateral unit cell (uc). Usually these electrons are localized in a Mott insulating state,[21] however it has been shown that high $\mu$ conduction can be generated if these electrons are released into a STO host. [2225] Here we investigate the use of LTO inserted at the LAO/STO interface to modulate the critical thickness, $n_{2 \mathrm{D}}$ and $\mu$.

All samples, with the structure sketched in the inset of Fig. 1(b), were prepared by pulsed laser deposition using a KrF excimer laser, grown on STO $\{100\}$ substrates with a $\mathrm{TiO}_{2}$ terminated surface. The substrates were preannealed at $1223 \mathrm{~K}$ for $30 \mathrm{mins}$ in an oxygen environment of $0.67 \mathrm{mPa}$. Following this anneal, the substrate temperature was reduced to $1023 \mathrm{~K}$ for LTO film growth, in a relatively low oxygen pressure $P_{\mathrm{O} 2}=0.133 \mathrm{mPa}$ to avoid formation of the $\mathrm{La}_{2} \mathrm{Ti}_{2} \mathrm{O}_{7}$ phase. [22, 23] The laser beam was imaged to form a spot of area $A=3.3 \times 1.8 \mathrm{~mm}^{2}$ on a polycrystalline $\mathrm{La}_{2} \mathrm{Ti}_{2} \mathrm{O}_{7}$ target using an afocal zoom stage; the total laser energy $E=26 \mathrm{~mJ}$, with a repetition rate $r$ of $4 \mathrm{~Hz}$. The LTO thickness, $y$, was varied in the range $0.25 \leq y \leq 4 \mathrm{uc}$, and controlled using in-situ reflection high-energy electron diffraction (RHEED). Clear RHEED intensity oscillations were observed for all samples with $y \geq 1$ uc. The growth rates were $\sim 40$ pulses/uc. For $y<1 \mathrm{uc}$, the thickness was controlled by laser pulse count, as calibrated from thicker samples.

After the growth of the LTO, the LAO was grown at $1073 \mathrm{~K}$ using a single crystal target, with $P_{\mathrm{O} 2}=$ $1.33 \mathrm{mPa}, A=2.3 \times 1.3 \mathrm{~mm}^{2}, E=20 \mathrm{~mJ}$, and $r=2 \mathrm{~Hz}$. The LAO thickness, $x$, was fixed at $x=$ 0, 1, 2, 3, 10 uc using RHEED oscillations, giving a growth rate of $\sim 45$ pulses/uc. The samples were cooled to room temperature at $P_{\mathrm{O} 2}=4 \times 10^{4} \mathrm{~Pa}$, with a one-hour pause at $873 \mathrm{~K} .[13] \mathrm{LAO}(x \mathrm{uc}) / \mathrm{LTO}(y \mathrm{uc}) / \mathrm{STO}$ samples, denoted by $(x, y)$ hereafter, were electrically contacted via ultrasonic wirebonding with aluminum wires, in a six-probe Hall bar configuration with voltage contacts $\sim 1 \mathrm{~mm}$ apart. All measurements were made with the sample normal parallel to the applied magnetic field. 


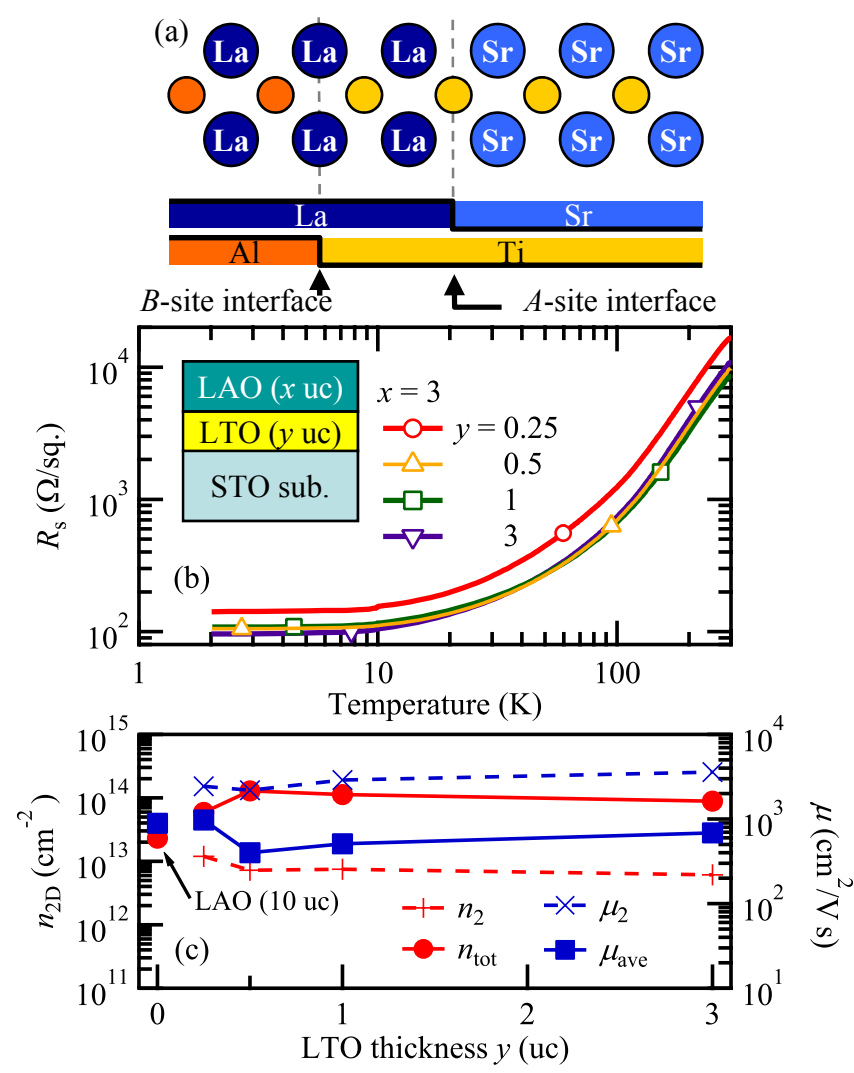

FIG. 1: (color online) (a) Schematic offset between the $A$-site interface $(\mathrm{La} \rightarrow \mathrm{Sr})$ and the $B$-site $(\mathrm{Al} \rightarrow \mathrm{Ti})$ due to the LTO insertion. (b) Temperature dependence of the sheet resistance and (c) the sheet carrier density and Hall mobility for representative samples with a LAO thickness of $3 \mathrm{uc},(3, y)$, at $T=2 \mathrm{~K}$. The inset of (b) shows a schematic of the structure of the samples. Data for LAO (10 uc)/STO from Ref. [17] are also plotted at $y=0$ in (c) for reference. Lines in (c) are guides.

The sample $(3,0)$ showed insulating behavior, while $(10,0)$ showed metallic behavior in agreement with the critical thickness previously reported.[13] All samples of the form $(10, y)$ and $(3, y)$ were also metallic for $y \geq 0.25$. Focusing on the $(3, y)$ series, the temperature dependence of the sheet resistances are shown in Fig. 1(b) for typical samples $(3,0.25),(3,0.5),(3,1)$ and $(3,3)$. All show a monotonically decreasing sheet resistance $R_{\mathrm{S}}$ with a residual sheet resistance of $\sim 100 \Omega$ /sq. at a temperature $T=2 \mathrm{~K}$. In the Hall resistance data, clear non-linearity was observed for all samples at low temperatures and magnetic fields up to $\mu_{0} H=8 \mathrm{~T}$. In the case of the $(3,0.25)$ sample, for example, the Hall co-efficient around $1 \mathrm{~T}(8 \mathrm{~T})$ was $-12.1(-10.2) \Omega \mathrm{T}^{-1}$. This non-linearity could be well fitted using a two-carrier model, constrained by the total sheet conductivity, where two parallel carrier layers with different sheet carrier densities and Hall mobilities are assumed, due to the spatial variation of the carrier density in the depth direction.[17, 23, 26]

The non-linear Hall effect fits give rise to layers with

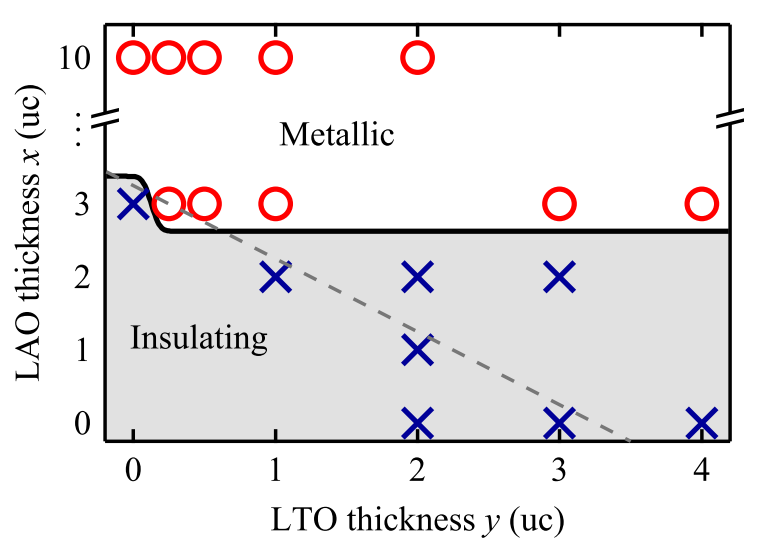

FIG. 2: (color online) Conductivity variation with different LAO and LTO thicknesses. Circles represent metallic conductivity, and crosses insulating behavior. Observed phase boundary is indicated with a solid line. Dashed line shows the predicted phase boundary $x+y \sim 3 \mathrm{uc}$ if the LTO is assumed to behave as a simple polar stack.

relatively high (low) sheet carrier density $n_{1}\left(n_{2}\right)$ and low (high) Hall mobility $\mu_{1}\left(\mu_{2}\right)$, representing carriers close to (far from) the interface, where scattering is relatively strong (weak), as shown in the inset of Fig. 3.[23] Fig. 1(c) shows the $y$ dependence of the total sheet carrier density $n_{\text {tot }}=n_{1}+n_{2}$, and averaged Hall mobility $\mu_{\text {ave }}=$ $\left(\mu_{1} n_{1}+\mu_{2} n_{2}\right) /\left(n_{1}+n_{2}\right)$ extracted from the two-carrier fit of the Hall resistance vs. $H$ at $T=2 \mathrm{~K}$, together with the $n_{2}$ and $\mu_{2}$ components. Clearly $n_{\text {tot }}$ rises rapidly at small $y$, reaching values of $n_{\text {tot }}=1.3 \times 10^{14} \mathrm{~cm}^{-2}$, before saturating above $y=0.5$ uc. For $x \leq 2$ uc, the samples were insulating independent of $y$. The character of the different $(x, y)$ samples is summarized in Fig. 2.

Combining these data, we can make important conclusions about the role of the LTO and LAO layers in controlling the interface conductivity. Firstly, we can consider LTO simply as a polar layer akin to LAO in the PD scenario, since the $\{100\}$ planes of LTO can be considered as alternating layers of $\left(\mathrm{La}^{3+} \mathrm{O}^{2-}\right)^{+}$and $\left(\mathrm{Ti}^{3+} \mathrm{O}_{2}^{4-}\right)^{-}$in the ionic limit. However this picture can be ruled out, since we would expect that conductivity is observed for $x+y \gtrsim 3$ uc, leading to a phase boundary shown by the dotted line in Fig. 2, in disagreement with the data. Instead we can consider LTO acting as a dopant layer due to the constituent $\mathrm{Ti}^{3+}$ ions, which form $3 d^{1}$ electrons that can migrate into the empty $3 d^{0}$ orbitals in the STO. It is clear that the saturation of $n_{\text {tot }}$ with increasing $y$ is inconsistent with a full electron per unit cell of LTO being released into the STO, given by $6.5 \times 10^{14} \mathrm{~cm}^{-2}$ per uc, although $n_{\text {tot }}$ is significantly higher than for typical LAO/STO samples without LTO.

When comparing the free carrier density with the nominal dopant value, related LTO/STO systems show a range of behaviors from significant reductions to densities 


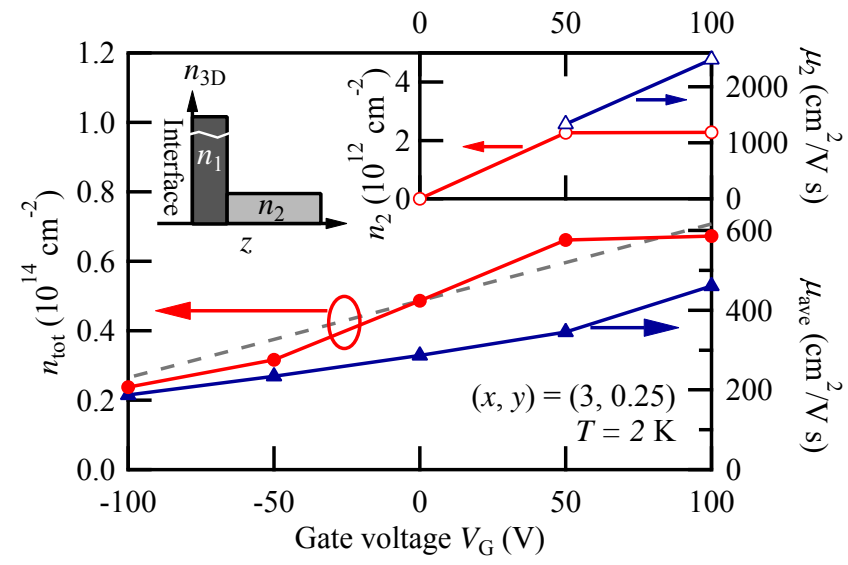

FIG. 3: (color online) $V_{\mathrm{G}}$ dependence of the sheet carrier density and Hall mobility of the sample $(3,0.25)$ extracted from a two-carrier fit at $T=2 \mathrm{~K}$. Dashed gray line shows the slope expected from the capacitance between the interface and gate contact; other lines are guides. Inset shows the schematic carrier distribution at the interface in the two-carrier model. $z$-axis points in the STO substrate direction.

of $\sim 10^{14} \mathrm{~cm}^{-2}$. [22-25] In our case $n_{2 \mathrm{D}}$ is at the larger end of that range, with moderate $\mu$. This may suggest that the LAO is also playing a role in determining the electron distribution in the STO. A simple interpretation is that the large conduction band offset between the STO and LAO, and the confining potential associated with the conducting interface produces a spatially more confined three dimensional carrier distribution in the direction perpendicular to the interface, $n_{3 \mathrm{D}}(z)$. This enhances electron overlap, which in a Mott criterion picture leads to less electron localization, and more activated carriers, as observed. Thus both the LTO and LAO layers may help to achieve these larger carrier densities, while maintaining mobilities $>300 \mathrm{~cm}^{2} / \mathrm{V}$ s at $T=2 \mathrm{~K}$. The observed ratio $n_{2} / n_{1} \sim 10^{-2}$, significantly smaller than observed in other LTO/STO heterostructures, $[23,25]$ can be understood as a consequence of the suppressed Hall mobility of the dense carriers close to the interface.

Next we investigated the effect of the LTO insertion on the field-effect response using a gate contact on the back side of the STO substrate. The gate voltage $V_{\mathrm{G}}$ dependences of the sheet carrier density and Hall mobility for a $(3,0.25)$ sample are shown in Fig. 3, with qualitatively similar results obtained for $(3,0.5)$ and $(3,1)$. Similar to previous results,[17] the Hall non-linearity was only observed for positive gate voltages, thus for $V_{\mathrm{G}}<0 \mathrm{~V}, n_{1}=n_{\text {tot }}$. For this $(3,0.25)$ sample, $n_{\text {tot }}=$ $4.9 \times 10^{13} \mathrm{~cm}^{-2}$ at $V_{\mathrm{G}}=0 \mathrm{~V}$ could be suppressed down to $2.4 \times 10^{13} \mathrm{~cm}^{-2}$ at $V_{\mathrm{G}}=-100 \mathrm{~V}$, in good agreement with the value expected from a simple capacitor model employing a STO dielectric constant $\varepsilon_{\mathrm{STO}}=20000$, and substrate thickness $0.5 \mathrm{~mm}$, as depicted as a dashed gray line in Fig. 3. For $V_{\mathrm{G}}>0$ both $n_{1}$ and $n_{2}$ increased,

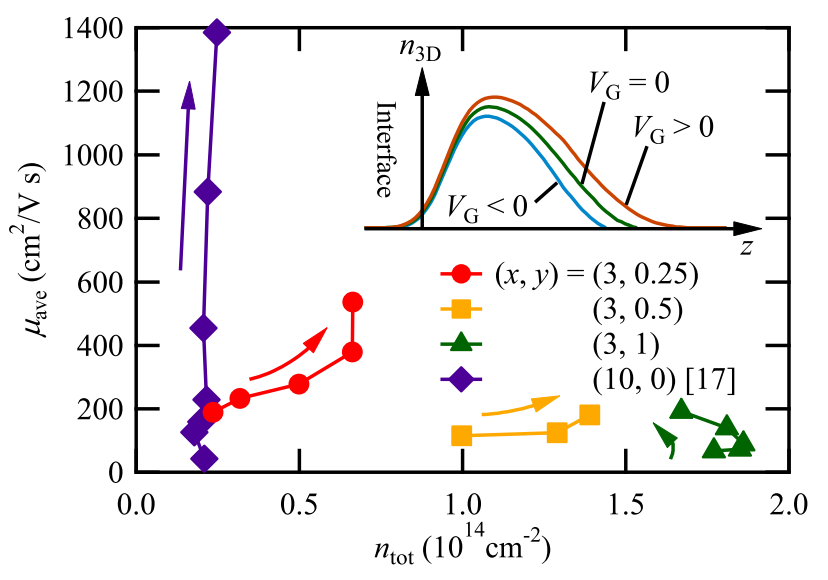

FIG. 4: (color online) Hall mobility vs. sheet carrier density achieved by back-gating several LAO/LTO/STO heterostructures at $T=2 \mathrm{~K}$. Arrows for each data set correspond to the direction of increasing $V_{\mathrm{G}}$ from $-100 \mathrm{~V}$ to $+100 \mathrm{~V}$. Lines are a guide. Inset shows schematic electron distribution along depth direction in $\mathrm{STO}, z$, for various $V_{\mathrm{G}}$

suggesting spreading of the carrier distribution into the depth of the substrate, where the Hall mobility is relatively enhanced. Interestingly, as shown in Fig. 4, the slope $\mathrm{d} \mu / \mathrm{d} n_{2 \mathrm{D}}$ for a previously studied LAO/STO sample is significantly larger than for the $(3,0.25)$ sample.

To understand these results, we note several important differences between samples with and without LTO. For $\mathrm{LAO} / \mathrm{STO}$, it is reasonable to assume that the confining electric field $E$ is determined self-consistently by the interfacial carrier density.[27] In the regime of a dense electron gas at the interface, a triangular potential well is a relatively good approximation, leading to an estimate of $E \sim 1.2 \times 10^{5} \mathrm{~V} / \mathrm{m}$ for $n_{2 \mathrm{D}}=2 \times 10^{13} \mathrm{~cm}^{-2}$.[17] On the other hand in the case of the LAO/LTO/STO systems, extra electrons from the LTO are introduced in the potential well, resulting in a much larger electric field at the interface. These electrons fill up to higher quantized eigenstates in the $z$ direction. One can then expect population or depopulation of electron gas via back gate can be achieved with a relatively smaller change in $E$. Upon reducing the positive electric field, the change in carrier distribution $n_{2} / n_{1}$ can be understood by considering the relatively large loss from the higher index eigenfunctions, having a peak in carrier density further from the interface, resulting in a significant decrease in $n_{2}$.

Finally we note that this ability to enhance the confined carrier density in the region of $10^{14} \mathrm{~cm}^{-2}$ may have important applications in the study of robust two-dimensional superconductivity in the ultra-thin limit, concomitant with signatures of Rashba spin-orbit coupling $[28,29]$ and magnetic order, also observed at high carrier density in other STO systems.[30] Indeed a priori the spin character of the electrons donated by 
the LTO layer is unclear,[31] providing further degrees of freedom at this fascinating interface.

The authors acknowledge support by the Department of Energy, Office of Basic Energy Sciences, Materials Sciences and Engineering Division, under contract DEAC02-76SF00515.

* Electronic address: mhosoda@slac.stanford.edu

[1] A. Ohtomo and H. Y. Hwang, Nature 427, 423 (2004).

[2] N. Reyren, S. Thiel, A. D. Caviglia, L. F. Kourkoutis, G. Hammerl, C. Richter, C. W. Schneider, T. Kopp, A.S. Ruetschi, D. Jaccard, M. Gabay, D. A. Muller, J.M. Triscone, and J. Mannhart, Science 317, 1196 (2007).

[3] A. D. Caviglia, S. Gariglio, C. Cancellieri, B. Sacépé, A. Fête, N. Reyren, M. Gabay, A. F. Morpurgo, and J.M. Triscone, Phys. Rev. Lett. 105, 236802 (2010).

[4] M. Ben Shalom, A. Ron, A. Palevski, and Y. Dagan, Phys. Rev. Lett. 105, 206401 (2010).

[5] J. A. Bert, B. Kalisky, C. Bell, M. Kim, Y. Hikita, H. Y. Hwang, and K. A. Moler, Nat. Phys. 7, 767 (2011).

[6] L. Li, C. Richter, J. Mannhart, and R. C. Ashoori, Nat. Phys. 7, 762 (2011).

[7] D. A. Dikin, M. Mehta, C. W. Bark, C. M. Folkman, C. B. Eom, and V. Chandrasekhar, Phys. Rev. Lett. 107, $056802(2011)$

[8] W. Siemons, G. Koster, H. Yamamoto, W. A. Harrison, G. Lucovsky, T. H. Geballe, D. H. A. Blank, and M. R. Beasley, Phys. Rev. Lett. 98, 196802 (2007).

[9] G. Herranz, M. Basletić, M. Bibes, C. Carrétéro, E. Tafra, E. Jacquet, K. Bouzehouane, C. Deranlot, A. Hamzić, J.-M. Broto, A. Barthélémy and A. Fert, Phys. Rev. Lett. 98, 216803 (2007).

[10] A. Brinkman, M. Huijben, M. van Zalk, J. Huijben, U. Zeitler, J. C. Maan, W. G. van der Wiel, G. Rijnders, D. H. A. Blank, and H. Hilgenkamp, Nat. Mater. 6, 493 (2007).

[11] P. R. Willmott, S. A. Pauli, R. Herger, C. M. Schlepütz, D. Martoccia, B. D. Patterson, B. Delley, R. Clarke, D. Kumah, C. Cionca, and Y. Yacoby, Phys. Rev. Lett. 99, 155502 (2007)

[12] N. Nakagawa, H. Y. Hwang, and D. A. Muller, Nat. Mater. 5, 204 (2006).

[13] S. Thiel, G. Hammerl, A. Schmehl, C. W. Schneider, and J. Mannhart, Science 313, 1942 (2006).

[14] D. G. Schlom and J. Mannhart, Nat. Mater. 10, 168
(2011).

[15] M. L. Reinle-Schmitt, C. Cancellieri, D. Li, D. Fontaine, M. Medarde, E. Pomjakushina, C. Schneider, S. Gariglio, Ph. Ghosez, J.-M. Triscone, and P. R. Willmott, Nat. Commun. 456, 624 (2012).

[16] A. D. Caviglia, S. Gariglio, N. Reyren, D. Jaccard, T. Schneider, M. Gabay, S. Thiel, G. Hammerl, J. Mannhart, and J.-M. Triscone, Nature 456, 624 (2008).

[17] C. Bell, S. Harashima, Y. Kozuka, M. Kim, B. G. Kim, Y. Hikita, and H. Y. Hwang, Phys. Rev. Lett. 103, 226802 (2009)

[18] T. Fix, J. L. MacManus-Driscoll, and M. G. Blamire, Appl. Phys. Lett. 94, 172101 (2009).

[19] W. Siemons, M. Huijben, G. Rijnders, D. H. A. Blank, T. H. Geballe, M. R. Beasley, and G. Koster, Phys. Rev. B 81, 241308 (2010).

[20] T. Fix, F. Schoofs, J. L. MacManus-Driscoll, and M. G. Blamire, Appl. Phys. Lett. 97, 072110 (2010).

[21] Y. Tokura, Y. Taguchi, Y. Okada, Y. Fujishima, T. Arima, K. Kumagai, and Y. Iye, Phys. Rev. Lett. 70, 2126 (1993).

[22] A. Ohtomo, D. A. Muller, J. L. Grazul, and H. Y. Hwang, Nature 419, 378 (2002).

[23] R. Ohtsuka, M. Matvejeff, K. Nishio, R. Takahashi, and M. Lippmaa, Appl. Phys. Lett. 96, 192111 (2010).

[24] J. Biscaras, N. Bergeal, A. Kushwaha, T. Wolf, A. Rastogi, R. Budhani, and J. Lesueur, Nat. Commun. 1, 89 (2010).

[25] J. Biscaras, N. Bergeal, S. Hurand, C. Grossetête, A. Rastogi, R. C. Budhani, D. LeBoeuf, C. Proust, and J. Lesueur, Phys. Rev. Lett. 108, 247004 (2012).

[26] J. S. Kim, S. S. A. Seo, M. F. Chisholm, R. K. Kremer, H.-U. Habermeier, B. Keimer, and H. N. Lee, Phys. Rev. B 82, 201407 (2010).

[27] F. Stern, Phys. Rev. B 5, 4891 (1972).

[28] M. Ben Shalom, M. Sachs, D. Rakhmilevitch, A. Palevski, and Y. Dagan, Phys. Rev. Lett. 104, 126802 (2010).

[29] A. D. Caviglia, M. Gabay, S. Gariglio, N. Reyren, C. Cancellieri, and J.-M. Triscone, Phys. Rev. Lett. 104, 126803 (2010).

[30] P. Moetakef, J. R. Williams, D. G. Ouellette, A. P. Kajdos, D. Goldhaber-Gordon, S. J. Allen, and S. Stemmer, Phys. Rev. X 2, 021014 (2012).

[31] S. Okamoto, A. J. Millis, and N. A. Spaldin, Phys. Rev. Lett. 97, 056802 (2006). 